\title{
QUALIDADE DAS ÁGUAS SUBTERRÂNEAS DE ÁREA IRRIGADA DA COMUNIDADE DE PAU BRANCO EM MOSSORÓ (RN)
}

\author{
N. S. Vasconcelos ${ }^{1,2}$, J. Dantas Neto ${ }^{2}$, J. F. de Medeiros ${ }^{3}$, C. J. G. S. Lima ${ }^{4}$ \\ ${ }^{1}$ Instituto Federal do Rio Grande do Norte, ${ }^{2}$ Programa de Pós-Grauduação em Recursos Naturais - Universidade \\ Federal de Campina Grande, ${ }^{3}$ Universidade Federal do Semi-Árido e ${ }^{4}$ Universidade Federal do Piauí \\ nelson.vasconcelos@ifrn.edu.br
}

Artigo submetido em fevereiro/2013 e aceito em março/2013

\begin{abstract}
RESUMO
A irrigação de culturas agrícolas sem o controle de alguns parâmetros de qualidade, associada ao uso indiscriminado de fertilizantes artificiais, tende a aumentar a poluição das águas superficiais e subterrâneas. Desse modo, o presente trabalho objetivou avaliar os níveis de qualidade da água subterrânea de uma propriedade agrícola de produção de melão irrigado, situada na comunidade de Pau Branco, em Mossoró-RN. Para a efetivação deste estudo, foram coletadas amostras de água de 13 poços tubulares, perfurados em uma área localizada no domínio de calcário Jandaíra, realizadas durante o ano de 2012. As amostras de água coletadas foram submetidas a algumas análises físicoquímicas de parâmetros de qualidade da água, de acordo com o método APHA (2005), destacando-se a condutividade elétrica; concentração de nitrato e potencial hidrogeniônico. Os dados foram analisados por meio de estatística descritiva, utilizando-se, da média (M), do desvio
\end{abstract}

padrão (DP), e dos valores mínimos e máximos desses dados. Para avaliar a precisão experimental dos dados, foi utilizado o coeficiente de variação (CV), conforme a classificação proposta por Pimentel-Gomes (1985). Foram empregados testes não paramétricos, visando ajustar os dados a uma distribuição normal com $5 \%$ de significância. Os parâmetros estudados, também, foram avaliados através dos gráficos de controle de qualidade de Shewhart. A partir da avaliação estatística, e de acordo com a classificação proposta por Ayers e Westcot (1999), constatou-se que os resultados, no período avaliado, dos valores de salinidade e de concentração de nitrato das águas desses poços foram classificados, quanto ao grau de restrição para uso, como de ligeiro a moderado. Os resultados dos valores médios de $\mathrm{pH}$ apresentaram limites aceitáveis, dentro de uma faixa normal, para utilização da água na irrigação, na cultura do melão.

PALAVRAS-CHAVE: Qualidade da água. Água subterrânea. Irrigação.

\section{QUALITY OF GROUNDWATER AREA IRRIGATED COMMUNITY OF PAU BRANCO IN MOSSORÓ}

\section{ABSTRACT}

Irrigation of agricultural crops without the control of some quality parameters, associated with the indiscriminate use of artificial fertilizers, tends to increase the pollution of surface and groundwater. However, the present study aimed to evaluate the quality of groundwater levels of an agricultural property, melon production irrigated, located in the community of PauBranco in Mossoró (RN). To accomplish this study, water samples were collected from 13 drilled tube wells in an area located in the limestone Jandaíra domain, held during the year of 2012. Water samples collected were subjected to some physicalchemical analysis of water quality parameters according to the APHA (2005), especially the electric conductivity; concentration of nitrate and potential hydrogen. Data were analyzed using descriptive statistics using, the mean (M) of the standard deviation (SD) and the minimum and maximum values of the data. To assess the accuracy of the experimental data, we used the coefficient of variation (CV), according to the classification proposed by PimentelGomes(1985).Were used non parametric tests in order to adjust the data to a normal distribution with $5 \%$ significance. The parameters studied were also evaluated by the quality control charts of Shewhart From this statistical assessment and according to the classification proposed by Ayers and Westcot (1999), it is noted that the results in the evaluated period, of the values of salinity and water nitrate concentration of these wells were classified as to the degree of restriction for use as mild to moderate. The results of the mean $\mathrm{pH}$ values exhibited acceptable limits within a normal range for use in irrigation water, culture melon.

KEY-WORDS: Water quality. Groundwater. Irrigation. 


\section{QUALIDADE DAS ÁGUAS SUBTERRÂNEAS DE ÁREA IRRIGADA DA COMUNIDADE DE PAU BRANCO EM MOSSORÓ (RN)}

\section{INTRODUÇÃO}

Em diversas regiões do Planeta, onde as precipitações são irregulares, a técnica da irrigação desempenha uma importante função na ampliação da produtividade agrícola, e, por conseguinte, na produção de alimentos. O aumento da demanda de recursos hídricos, com a expansão das áreas irrigadas,tem sido objeto de constante preocupação, quando se almeja o desenvolvimento sustentável aliado ao uso racional de recursos naturais(REIS et al., 2011).

A prática da irrigação, sem os controles necessários, associada ao regime irregular das chuvas e às elevadas taxas de evaporação nas regiões semiáridas, tende a aumentar os teores de sais nos solos e nas águas. Devido a fatores climáticos, a condições edáficas e aos métodos de irrigação, os sais dissolvidos na água podem se acumular no perfil do solo ou serem carreados para as águas subterrâneas (RODRIGUES et al., 2007; ANDRADE et al., 2009).

A implantação da irrigação tem promovido, em larga escala, mudanças no regime hidrológico local, resultando no acúmulo de sais na superfície do solo, ascensão do lençol freático, depleção dos aquíferos e contaminação dos recursos hídricos (ANDRADE, 2009).

O acúmulo de sais solúveis, além de reduzir o potencial osmótico da solução do solo, produz alteração no pH,desbalanceamento nutricional e desestruturação de seus agregados (MEDEIROS et al., 2010).

Para se conseguir altas produtividades agrícolas, se tem combinado a técnica da irrigação com o uso indiscriminado dos fertilizantes químicos. A produção de fertilizantes artificiais e sua intensificação na agricultura globalizada têm incrementado o surgimento de nitrogênio, em ecossistemas aquáticos e terrestres, numa ordem de magnitude nunca vista desde a revolução industrial (GALLOWAY et al., 2008).Diante dessa constatação e, segundo García-Garizábalet al. (2012), a contaminação de nitrato tem se tornado o maior problema para a agricultura deste século.

O íon nitrato $\left(\mathrm{NO}_{3}{ }^{-}\right)$não é adsorvido pelos componentes das frações do solo, razão pela qual se desloca facilmente na solução do solo, podendo ser absorvido pelas raízes e translocado às folhas, onde se acumula pela transpiração, ou serlixiviado aos mananciais subterrâneos (AYRES; WESTCOST, 1999).

Os recursos hídricos agem como integradores dos processos biogeoquímicos de qualquer área ou região. Nesse sentido, quando água e fertilizantes em excesso são aplicados, particularmente na agricultura, os recursos hídricos, sejam superficiais ou subterrâneos, são os principais destinos a serem atingidos. Por isso, a preocupação com a qualidade da água usada na irrigação tem sido objeto de pesquisa nos últimos anos, e a sua classificação de fundamental importância, uma vez quefornece subsídios para a avaliação da sua utilização em relação ao sistema água-solo-planta e ao regime de irrigação. Para Srinivasanet al. (2009), a qualidade da água afeta um sistema de irrigação desde a cultura até as propriedades do solo.Por outro lado, Hassanliet al.(2009) afirmam que o uso da água de boa qualidade em lavoura irrigada promove a agricultura sustentável, já quedesenvolvimento sustentável e agricultura desempenham um papel importante na questão da segurança alimentar. 
No Estado do Rio Grande do Norte, grande parte da água usada na área produtora de melão tem sido proveniente de poços tubulares abertos no calcário Jandaíra, em virtude da sua maior disponibilidade hídrica.

Essas fontes de água, perfuradas no aqüíferoJandaíra, apresentam níveis elevados de sais, podendo causar a salinização dos solos e prejudicar o rendimento das culturas (MEDEIROS et al., 2003). A salinidade dessas águas às vezes supera2000 $\mathrm{mg} \mathrm{L}^{-1}$, aproximadamente $3,0 \mathrm{dS} \cdot \mathrm{m}^{-1}$ (OLIVEIRA; MAIA,1998). Nesse caso, a utilização da água fica condicionada à tolerância das culturas, à salinidade e ao manejo da irrigação, com vistas ao controle da salinização da área de produção.

O aqüíferoJandaíra, face às suas características cárstico-fraturada, e comportando-se como aqüíferolivre, apresenta elevada vulnerabilidade quanto aos riscos de contaminação proporcionados pela agricultura intensiva da região, diante do uso indiscriminado de fertilizantes.

Geologicamente, o município de Mossoróencontra-se, na Província de Borborema, sendo constituído de rochas carbonáticas da Formação Jandaíra e do Grupo Barreiras. Ele está inserido no Domínio Hidrogeológico Intersticial e Karstico-fissural. Os aquíferos nesse domínio são vulneráveis à poluição, isto ocorre porque os sistemas de fissuras das rochas calcárias facilitam a rápida penetração dos poluentes no aquífero (FRISTZSONSet al. 2001). As regiões cársticas normalmente são áreas de grande interesse econômico e hidrogeológico porque, na maioria das vezes, possuem bons solos agricultáveis, não apresentam drenagem superficial, possuem valiosas reservas de água no subsolo e têm grande importância ambiental (SILVA, 2012). Em relação aos aspectos socioeconômicos, a Região se destaca no campo da produção agrícola pela importância da fruticultura irrigada, com ênfase para a cultura do melão no desenvolvimento da economia regional, implicando em retorno social e ocupando uma posição de destaque no cenário internacional na exportação de frutas tropicais.

Para um manejo adequado do uso racional da água de irrigação, um monitoramento da qualidade da água, realizado por meio de estudos de campo bem planejados, pode gerar informações que subsidiem ações para minimizar a contaminação das águas subterrâneas na região de Mossoró, produtora de melão por irrigação, no calcário Jandaíra.

O presente trabalho teve por objetivo avaliar a qualidade da água utilizada para fins de irrigação, procedente de treze poços tubulares da fazenda Dinamarca, produtora de melão irrigado, situada na comunidade de Pau Branco, na região de Mossoró.

\section{MATERIAIS E MÉTODOS}

O experimento de campo foi realizado no período de 12 de janeiro a 25 de outubro de 2012, na fazenda Dinamarca Industrial Agrícola LTDA, produtora de melão pelo processo da fertirrigação. A fazenda está situada na comunidade Rural de Pau Branco, distante $30 \mathrm{~km}$ da sede municipal de Mossoró (RN), cujas coordenadas são: $4^{\circ} 54^{\prime} 28^{\prime \prime} \mathrm{S}$ e $37^{\circ} 24^{\prime} 06^{\prime \prime} \mathrm{O}$, ocupando uma área de 552 hectares(Figura 1). O clima da região, de acordo com a classificação climática de Köppen, é do grupo BSwh', caracterizado por ser quente e seco; com precipitações pluviométricas bastante irregulares, média anual de $675,8 \mathrm{~mm}$, período chuvoso somente de fevereiro a abril, temperatura média anual em torno de $27 \stackrel{\circ}{\circ}$ e umidade relativa média anual de 69\% (CARMO FILHO; OLIVEIRA,1995). 
Figura 1 - Localização geográfica e imagem via satélite dos pontos de coleta de amostras de água dos poços tubulares na área de estudo na fazenda Dinamarca em Mossoró - RN, 2013.

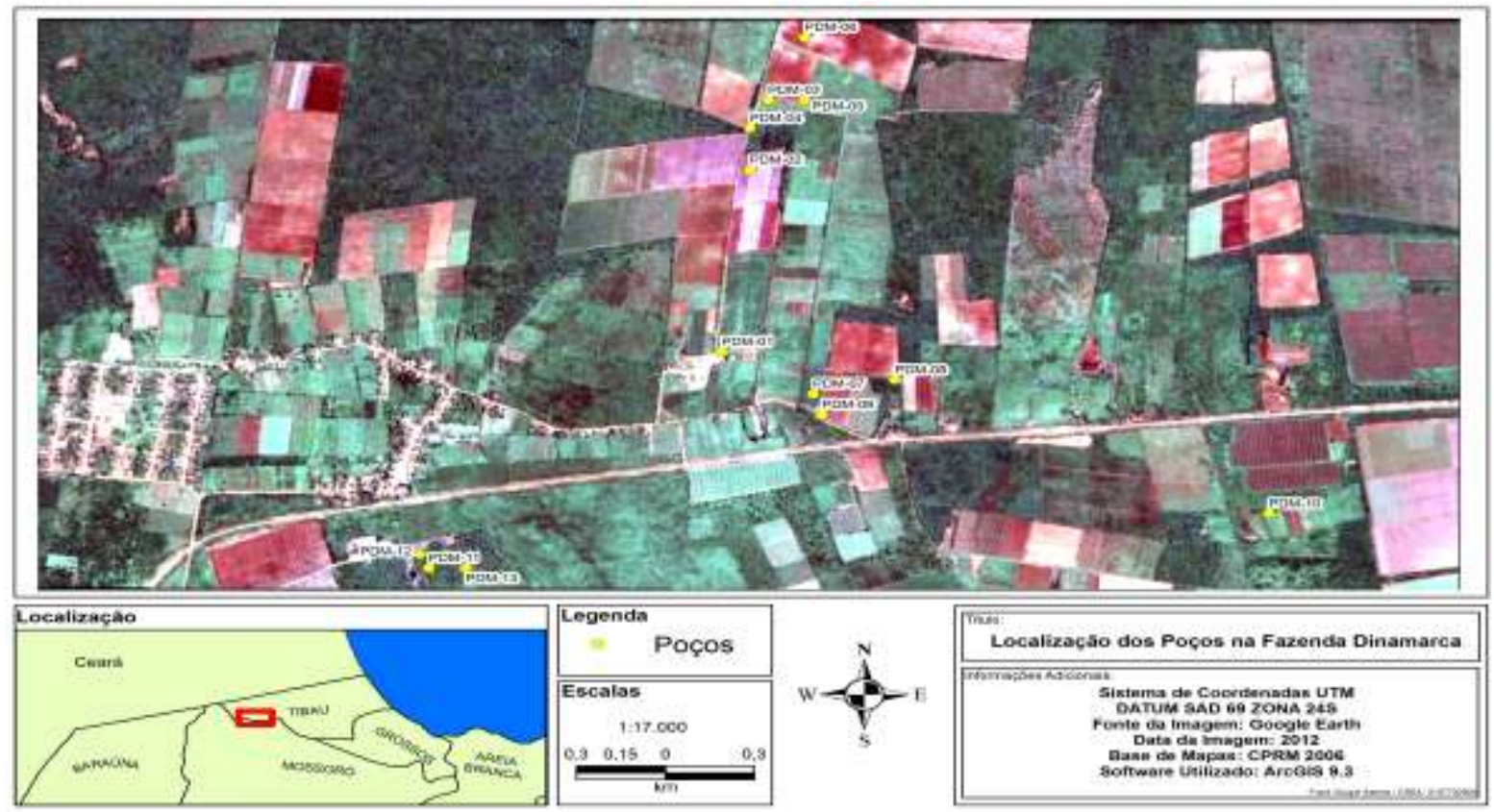

Fonte: Google Earth (2012).

$\mathrm{Na}$ área de estudo foram selecionados e georreferenciados, 13 poços tubulares (Figura 1) perfurados no aqüíferoJandaíra, com profundidade média de aproximadamente $58 \mathrm{~m}$, para o monitoramento de alguns parâmetros de qualidade da água no ano de 2012. As características de cada poço são apresentadas na tabela 1. As coletas de amostras de água foram realizadas entre os meses de janeiro, março, maio, junho, julho, setembro e outubro de 2012, com um total de 91 amostras. Durante esse período a precipitação acumulada da área foi de 223,2 mm, segundo levantamentosclimáticos da EMPARN (2012)

Tabela 1 - Características dos poços selecionados da fazenda Dinamarca. Ano 2012

\begin{tabular}{|c|c|c|c|c|c|}
\hline Poço & $\begin{array}{l}\text { Coordenadas } \\
\text { geográficas }\end{array}$ & $\begin{array}{c}\text { Profundidade } \\
(\mathrm{m})\end{array}$ & $\begin{array}{l}\text { Vazão } \\
\left(\mathrm{m}^{3} / \mathrm{h}\right)\end{array}$ & $\begin{array}{l}\text { Potência da } \\
\text { bomba (CV) }\end{array}$ & $\begin{array}{c}\text { Diâmetro } \\
\text { poço (pol.) }\end{array}$ \\
\hline & $4^{\circ} 54^{\prime} 26.42^{\prime \prime} \mathrm{S}$ & & & & \\
\hline PDM-01 & $\begin{array}{c}37^{\circ} 24^{\prime} 04.08^{\prime \prime} \mathrm{O} \\
\text { Elev. } 17 \mathrm{~m}\end{array}$ & 31 & 50,4 & 15 & 8 \\
\hline PDM-02 & $\begin{array}{c}4^{\circ} 53^{\prime} 59.77^{\prime \prime} \mathrm{S} \\
37^{\circ} 24^{\prime} 01.40^{\prime \prime} \mathrm{O} \\
\text { Elev. } 28 \mathrm{~m} \\
4^{\circ} 53^{\prime} 49.68^{\prime \prime} \mathrm{S}\end{array}$ & 68 & 99 & 25 & 8 \\
\hline PDM-03 & $\begin{array}{c}37^{\circ} 23^{\prime} 59.23^{\prime \prime} \mathrm{O} \\
\text { Elev. } 32 \mathrm{~m} \\
4^{\circ} 53^{\prime} 54.33^{\prime \prime} \mathrm{S}\end{array}$ & 68 & 95,2 & 25 & 8 \\
\hline PDM-04 & $\begin{array}{c}37^{\circ} 24^{\prime} 00.51^{\prime \prime} \mathrm{O} \\
\text { Elev. } 30 \mathrm{~m} \\
4^{\circ} 53^{\prime} 49.98^{\prime \prime} \mathrm{S}\end{array}$ & 70 & 26 & 8 & 8 \\
\hline
\end{tabular}




\begin{tabular}{|c|c|c|c|c|c|}
\hline PDM-05 & $\begin{array}{c}37^{\circ} 23^{\prime} 55.06^{\prime \prime} \mathrm{O} \\
\text { Elev. } 35 \mathrm{~m} \\
4^{\circ} 53^{\prime} 41.24^{\prime \prime} \mathrm{S}\end{array}$ & 69 & 60 & 20 & 8 \\
\hline PDM-06 & $\begin{array}{c}37^{\circ} 23^{\prime} 55.15^{\prime \prime} \mathrm{O} \\
\text { Elev. } 35 \mathrm{~m} \\
4^{\circ} 54^{\prime} 32.28^{\prime \prime} \mathrm{S}\end{array}$ & 50 & 60 & 20 & 8 \\
\hline PDM-07 & $\begin{array}{c}37^{\circ} 23^{\prime} 54.07^{\prime \prime} \mathrm{O} \\
\text { Elev. } 20 \mathrm{~m} \\
4^{\circ} 54^{\prime} 29.69^{\prime \prime} \mathrm{S}\end{array}$ & 50 & 60 & 20 & 8 \\
\hline PDM-08 & $\begin{array}{c}37^{\circ} 23^{\prime} 44.71^{\prime \prime} \mathrm{O} \\
\text { Elev. } 24 \mathrm{~m} \\
4^{\circ} 54^{\prime} 35.01^{\prime \prime} \mathrm{S}\end{array}$ & 52 & 28 & 80 & 6 \\
\hline PDM-09 & $\begin{array}{c}37^{\circ} 23^{\prime} 53.25^{\prime \prime} \mathrm{O} \\
\text { Elev. } 20 \mathrm{~m} \\
4^{\circ} 54^{\prime} 49.46^{\prime \prime} \mathrm{S}\end{array}$ & 50 & 60 & 20 & 8 \\
\hline PDM-10 & $\begin{array}{c}37^{\circ} 23^{\prime} 03.66^{\prime \prime} \mathrm{O} \\
\text { Elev. } 20 \mathrm{~m} \\
4^{\circ} 54^{\prime} 57.33^{\prime \prime} \mathrm{S}\end{array}$ & 60 & 50 & 20 & 8 \\
\hline PDM-11 & $\begin{array}{c}37^{\circ} 24^{\prime} 36.24^{\prime \prime} \mathrm{O} \\
\text { Elev. } 23 \mathrm{~m} \\
4^{\circ} 54^{\prime} 55.28^{\prime \prime} \mathrm{S}\end{array}$ & 55 & 60 & 20 & 8 \\
\hline PDM-12 & $\begin{array}{c}37^{\circ} 24^{\prime} 36.98^{\prime \prime} \mathrm{O} \\
\text { Elev. } 23 \mathrm{~m} \\
4^{\circ} 54^{\prime} 56.80^{\prime \prime} \mathrm{S}\end{array}$ & 60 & 55 & 25 & 8 \\
\hline PDM-13 & $\begin{array}{c}37^{\circ} 24^{\prime} 31.88^{\prime \prime} \mathrm{O} \\
\text { Elev. } 23 \mathrm{~m}\end{array}$ & 75 & 60 & 25 & 8 \\
\hline
\end{tabular}

Fonte: Os autores (2012)

\section{PARÂMETROS AVALIADOS}

Os parâmetros selecionados para avaliar os níveis de qualidade da água seguiram as recomendações Ayers e Westcot (1999), sendo estes: condutividade elétrica (CE), nitrato ( $\mathrm{NO}_{3}{ }^{-}$$\mathrm{N})$ e potencial hidrogeniônico $(\mathrm{pH})$. Todos os procedimentos de análise foram realizados de acordo com os métodos da APHA (2005) e os critériosde coleta seguiram a NBR 9898/87. As analises dos teores de nitrato foram realizadas no Laboratório de química do IFRN, Natal-Central. Os valores de $\mathrm{pH}$ e condutividade elétrica foram medidos em campo na saída da água da tubulação da bomba, com o auxílio de phgâmetros e condutivimetros portáteis.

Os dados foram analisados por meio de estatística descritiva, utilizando-se, da média (M) do desvio padrão (DP), e dos valores mínimos e máximos dos dados. Para avaliar a precisão experimental dos dados, dos diferentes parâmetros, foi utilizado o coeficiente de variação (CV). De acordo com os dados experimentais, a classificação dos coeficientes de variaçãofoi determinada a partir de intervalos definidos, conforme os propostos por Pimentel-Gomes (1985), como mostra a tabela 2. 
Tabela 2 - Classificação e intervalos dos coeficientes de variação

\begin{tabular}{cc}
\hline $\begin{array}{c}\text { Classificação dos coeficientes de } \\
\text { variação (CV) }\end{array}$ & Intervalos dos coeficientes de variação \\
\hline Baixo & $\mathrm{CV} \leq 10 \%$ \\
Médio & $10 \%<\mathrm{CV} \leq 20 \%$ \\
Alto & $20 \%<\mathrm{CV} \leq 30 \%$ \\
Muito alto & $\mathrm{CV}>30 \%$ \\
\hline
\end{tabular}

Fonte: Pimentel-Gomes (1985).

Além disso, procurou-se ajustar os dados à distribuição normal com $5 \%$ de significância utilizando-se dealguns testes não-paramétricos de normalidade, tais como: Kolmogorov-Smirnov, com a modificação de Lilliefors(1967),Cramér-Von Mises, Anderson-Darling, Kuiper, Watson, Lilliefors e Shapiro- Wilk.

Foram utilizados os limites de tolerância de cada parâmetro, estabelecidos porAyers e Westcot (1999), para a utilização da água na irrigação, caracterizando quantoàs restrições ao uso.

A partir dos resultados das análises físico-químicas, obtidos pelos parâmetros analisados, procedeu-se uma avaliação do controle de qualidade das amostras de água, baseada nos gráficos de controle de Shewhart, que são ferramentas eficientes no monitoramento de qualidade de um processo e no atendimento às Normas de qualidade; ademais, é também utilizado para identificar quando um processo encontra-se fora de controle.Esse gráfico é representado por uma linha Central (LC), ou linha média (LM), que representa o valor médio da característica de qualidade e duas outras linhas horizontais denominadas, respectivamente, de Limite Superior de Controle (LSC) e Limite Inferior de Controle (LIC). Os dois limites ficam a uma distância de três desvios-padrão da média $(\mu \pm 3 \sigma$ ), de acordo com Shewhart (1931). Pontos amostrais dentro dos limites de controle equivalem a não rejeitar a hipótese de que o processo está sob controle estatístico e pontos fora dos limites equivalem a rejeitar esta hipótese, conforme (MONTGOMERY, 2004).

Baseando-se no número de observações foramutilizadas duas regras de controle, a saber:a) $\mathbf{1}_{2 s}$ representando a regra de controle na qual uma observação de controle excede os limites de controle $x \pm 2 \mathrm{DP}$. Esta é uma regra de alertapresente no gráfico de Shewhart e é interpretada nessa discussão como uma exigência para uma inspeção adicional dos resultados de controle, testando os resultados com a regra seguinte para julgar quando a corrida analítica deve ser aceita ou rejeitada;b) $\mathbf{1}_{3 s}$,simbolizando a regra de controle na qual uma corrida é rejeitada, quando uma observação de controle excede o limite $x \pm 3 D P$. Esses são os limites de rejeição usuais em um gráfico de controle de Shewhart, em que xrepresentaa média e DP corresponde ao desvio padrão dos dados da amostra.

\section{RESULTADOS E DISCUSSÃO}

\section{Testes de Normalidade}

A maioria dos métodos estatísticos usuaisparte da hipótese de que os dados apresentem uma distribuição de probabilidade Normal.Baseado nessa suposição de normalidade dos dados, é de fundamental importância comprovar a sua veracidade, através de alguns testes não-paramétricos de normalidade, tais como: Kolmogorov-Smirnov, com a modificação de Lilliefors; , Cramér-Von Mises, 
Anderson-Darling, Kuiper, Watson, Lilliefors e Shapiro- Wilk. Os testesforam aplicados na avaliação dos parâmetros de $\mathrm{pH}$, condutividade elétrica e nitrato das 91 amostras de água coletadas em poços tubulares da fazenda Dinamarca, no período de janeiro a outubro de 2012. A aplicação dos testes foi através doSoftware Assistat-Statistical 7.6 beta ( SILVA,2009)

A tabela 3mostra os resultados daaplicação do teste de normalidade KolmogorovSmirnov, com a modificação de Lilliefors (LILLIEFORS 1967).Segundo a análise dos resultados, somente o parâmetro nitrato $(p<0,15)$ apresentou distribuição normal com $5 \%$ de significância.

Tabela 3 - Estatística descritiva dos parâmetros avaliados conforme teste de normalidade Kolmogorov-Smirnov, com a modificação de Lilliefors (1967)

\begin{tabular}{|c|c|c|c|c|c|c|c|c|c|}
\hline \multirow{3}{*}{ Parâmetro } & \multirow{3}{*}{$\begin{array}{l}\text { Valor } \\
\text { Max. }\end{array}$} & \multirow{3}{*}{$\begin{array}{l}\text { Valor } \\
\text { Mín. }\end{array}$} & \multicolumn{3}{|c|}{ Desvio } & \multicolumn{4}{|c|}{ Normalidade a $5 \%$} \\
\hline & & & Média & & CV (\%) & & & & \\
\hline & & & & Padrão & & Valor & Vcrit & p-valor & Normal \\
\hline $\mathrm{CE}\left(\mathrm{dS} \mathrm{m^{-1 } )}\right.$ & 2,92 & 2,00 & 2,37 & 0,15 & 6,33 & 0.11 & 0.09 & $p<.01$ & Não \\
\hline $\mathrm{N}-\mathrm{NO}_{3}{ }^{-}\left(\mu \mathrm{g} \cdot \mathrm{L}^{-1}\right) \cdot 10^{3}$ & 17,15 & 10,07 & 13,51 & 1,49 & 11,00 & 0.07 & 0.09 & $p<.15$ & Sim \\
\hline pH & 7,71 & 7,14 & 7,40 & 0,13 & 1,71 & 0.21 & 0.09 & $p<.01$ & Não \\
\hline
\end{tabular}

Fonte: Os autores (2012)

Nota: p-valor : nível de significância observado.

Vcrit: valor crítico

As tabelas 4,5 e 6 sintetizam a aplicação e os resultados dos seguintes testes de normalidade, destacando-se, os seguintes: Kolmogorov-Smirnov, Cramér-Von Mises, AndersonDarling, Kuiper, Watson, Lilliefors e Shapiro- Wilk.Cada um desses testes apresentam critérios metodológicos diferentes para a comprovação da veracidade do teste de hipóteses da normalidade dos dados.

Segundo as análises dos testes estatísticos de normalidade, para as variáveis condutividade elétrica (CE), nitrato $\left(\mathrm{NO}_{3}{ }^{-}-\mathrm{N}\right)$ e $\mathrm{pH}$, somente o parâmetro nitrato apresentou distribuição normal com $5 \%$ de significância (Tabela 5).

Tabela 4-Estatística descritiva dos resultadosde alguns testes de normalidade a 5\% para a Condutividade elétrica ( CE), das 91 amostras de água coletadas nos poços da área de estudo (Janeiro a outubro de 2012)

\begin{tabular}{ccccc}
\hline Teste & Valor & VCritico & $\mathrm{p}$-valor & Normal \\
\hline Kolmogorov-Smirnov & 0.11109 & 0.09305 & $\mathrm{P}<.01$ & Não \\
Cramér-Von Mises & 0.23128 & 0.12531 & $\mathrm{P}<.01$ & Não \\
Anderson-Darling & 1.52824 & 0.75605 & $\mathrm{P}<.01$ & Não \\
Kuiper & 0.20987 & 0.15390 & $\mathrm{P}<.01$ & Não \\
Watson & 0.19681 & 0.11537 & $\mathrm{P}<.01$ & Não \\
Lilliefors & 0,11109 & 0.09288 & $\mathrm{P}<.01$ & Não \\
Shapiro- Wilk & 0.94162 & - & 0.00049 & Não \\
\hline
\end{tabular}

Fonte: $\mathrm{O}$ autor 
Tabela 5- Estatística descritiva dos resultadosde alguns testes de normalidade a 5\% para o nitrato $\left(\mathrm{NO}_{3}{ }^{-}-\mathrm{N}\right)$, das 91 amostras de água coletadas nos poços da área de estudo (Janeiro a outubro de 2012)

\begin{tabular}{ccccc}
\hline Teste & Valor & V Critico & p-valor & Normal \\
\hline Kolmogorov-Smirnov & 0.07839 & 0.09305 & $\mathrm{P}<.15$ & $\mathrm{Sim}$ \\
Cramér-Von Mises & 0.12055 & 0.12531 & $\mathrm{P}<.05$ & $\mathrm{Sim}$ \\
Anderson-Darling & 0.67301 & 0.75605 & $\mathrm{P}<.05$ & $\mathrm{Sim}$ \\
Kuiper & 0.13677 & 0.15390 & $\mathrm{P}<.10$ & $\mathrm{Sim}$ \\
Watson & 0.11367 & 0.11537 & $\mathrm{P}<.05$ & $\mathrm{Sim}$ \\
Lilliefors & 0,07839 & 0.09288 & $\mathrm{P}<.15$ & $\mathrm{Sim}$ \\
Shapiro- Wilk & 0.98182 & - & 0.23483 & $\mathrm{Sim}$ \\
\hline
\end{tabular}

Fonte: $O$ autor

Tabela 6 - Estatística descritiva dos resultados de alguns testes de normalidade a 5\% para pH das 91 amostras de água coletadas nos poços da área de estudo (Janeiro a outubro de 2012)

\begin{tabular}{ccccc}
\hline Teste & Valor & V Critico & $\mathbf{p}$-valor & Normal \\
\hline Kolmogorov-Smirnov & 0.21789 & 0.09305 & $\mathrm{P}<.01$ & Não \\
Cramér-Von Mises & 0.60065 & 0.12531 & $\mathrm{P}<.01$ & Não \\
Anderson-Darling & 3.32513 & 0.75605 & $\mathrm{P}<.01$ & Não \\
Kuiper & 0.31839 & 0.15390 & $\mathrm{P}<.01$ & Não \\
Watson & 0.58807 & 0.11537 & $\mathrm{P}<.01$ & Não \\
Lilliefors & 0.21789 & 0.09288 & $\mathrm{P}<.01$ & Não \\
Shapiro- Wilk & 0.92459 & - & 0.00006 & Não \\
\hline
\end{tabular}

Fonte: $\mathrm{O}$ autor

\section{CONDUTIVIDADE ELÉTRICA}

A Condutividade elétrica (CE) mede a capacidade que a água tem de conduzir corrente elétrica, possuindo uma relação direta com as espécies iônicas dissolvidas. Quanto maior forem à quantidade de íons dissolvidos maiores os valores de CE. Devido à facilidade na sua determinação, é o parâmetro considerado para estabelecer a sua potencialidade de salinizar um solo. Os valores de CE nos poços da fazenda Dinamarca variaram de 2,92 a 2,00 (dS m ${ }^{-1}$ ), com valor médio de 2,37 ( $\mathrm{dS} \mathrm{m}^{-1}$ ), conforme a Tabela 3. O coeficiente de variação dos dados foi de 6,33\%, sendo considerado baixo, conforme Pimentel-Gomes (1985). A classificação do CV é inversamente proporcional à classificaçãoda precisão do experimento, ou seja, quanto maior o CV menor a precisãoexperimental. Deste modo, o baixo valor de CV dos dados de CE representa uma pequena variabilidade desse parâmetro e alta precisão experimental.

Os valores de salinidade da água dos poços da fazenda Dinamarca, expressos em CE, encontrados nessa pesquisa, estão coerentes com os resultados de Marouelliet al. (2003). Esses autores afirmam que as águas do aquífero calcário Jandaira, utilizada para produzir melão, nessa região, apresentam concentrações de sais relativamente elevadas, podendo ser superior a 2000

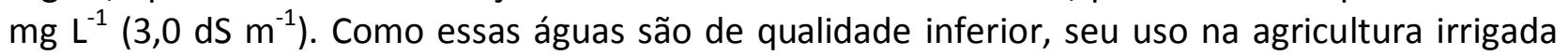
requer adoção de um manejo mais criterioso, de maneira que, além da aplicação de lâminas de irrigação e níveis de umidade adequados no solo, sua utilização fica condicionada à tolerância das 
culturas à salinidade, de forma a se obter altas produtividades com frutos de boa qualidade e com mínimos riscos de salinização dos solos.

Seguindo as diretrizes, para interpretação da qualidade das águas para irrigação propostas por Ayers e Westcot (1999), a salinidade das águas dos poços da fazenda Dinamarca expressos em CE (Tabela 3), possuem um grau de restrição de uso dessa água, de ligeiro a moderado.

Entende-se que valores de condutividade acima de $0,7 \mathrm{dS} \mathrm{m}^{-1}$ podem afetar a disponibilidade de água para algumas culturas. Estudos realizados por Medeiros et al. (2000), com o hibrido de melão amarelo Gold mine, mostraram que a aplicação de água com condutividade elétrica (CEa) de $2,65 \mathrm{dS} \mathrm{m}^{-1}$ reduziu a produtividade da cultura em $27 \%$ e o número de frutos em $19 \%$ em relação à irrigação com água com condutividade elétrica de 0,55 dS $\mathrm{m}^{-1}$. O melão amarelo, cultivar AF646, sofreu redução linear em sua produção quando a salinidade da água variou de 0,6 para $4,5 \mathrm{dS} \mathrm{m}^{-1}$, tendo sido observado que, para cada acréscimo de $1 \mathrm{dS} \mathrm{m}^{-1}$ na CEa, houve uma redução de cerca de 9,5\% no número de frutos comerciais e de $10 \%$ na produção comercial (SOUZA NETO , 2003).

A Figura 2 apresenta os valores médios da condutividade elétrica (CE) de amostras de água, coletada e analisada durante o ano de 2012 .O máximo valor médio de CE foi encontrado no mês outubro, 2,54dS $\mathrm{m}^{-1}$, época de maior demanda de água da cultura do melão, consequentemente maior lixiviação de corretivos e fertilizantes. O menor valor de CE foi encontrado no mês de março provavelmente pela diluição provocada pelas precipitações ocorridas em fevereiro de 2012.

Em regiões onde a precipitação total anual é superior a $800 \mathrm{~mm} \mathrm{ano}^{-1}$, a lixiviação dos sais passa a ser determinada pela textura e estrutura do solo (ANDRADE, 2009). Em solos arenosos, na região litorânea do Ceará, irrigados por aspersão, foi identificado que $400 \mathrm{~mm}$ ano-1 era suficiente para promover a lixiviação dos sais adicionados ao solo pela prática da irrigação (PEREIRA et al.,1986). Em solos de textura mais pesada (franco-argiloso), Ben-Huret al. (2001) verificaram que a precipitação anual de $600 \mathrm{~mm}$ promoveu a lixiviação de sais, em um Vertisol, na região do Yizre'el Valley, em Israel. Já nas áreas irrigadas do Distrito de Irrigação JaguaribeApodi (DIJA) localizado no Baixo Jaguaribe (CE), Andrade (2009) relata acúmulo de sais no perfil do solo, mesmo em precipitações anuais superiores a $750 \mathrm{~mm}$.

A alta variabilidade no valor da CE entre estações secas e chuvosas também foi observada por Franca et al. (2006), ao analisarem a qualidade das águas de uma bateria de poços tubulares, que abastece o município de Juazeiro do Norte, no estado do Ceará (CE). Durante o período seco, a condutividade elétrica média da bateria de poços estudados variou de $0,150 \mathrm{dS} \mathrm{m}^{-1}$, a 0,549 dS $\mathrm{m}^{-1}$. Já durante o período chuvoso, a CE variou entre $0,131 \mathrm{dS} \mathrm{m}^{-1}$ e $0,506 \mathrm{dS} \mathrm{m}^{-1}$, no entanto, no período chuvoso ocorreu uma diminuição dos valores em quase todos os poços. 
Figura 2 - Valores médios de condutividade elétrica, CE, ao longo do ano de 2012.

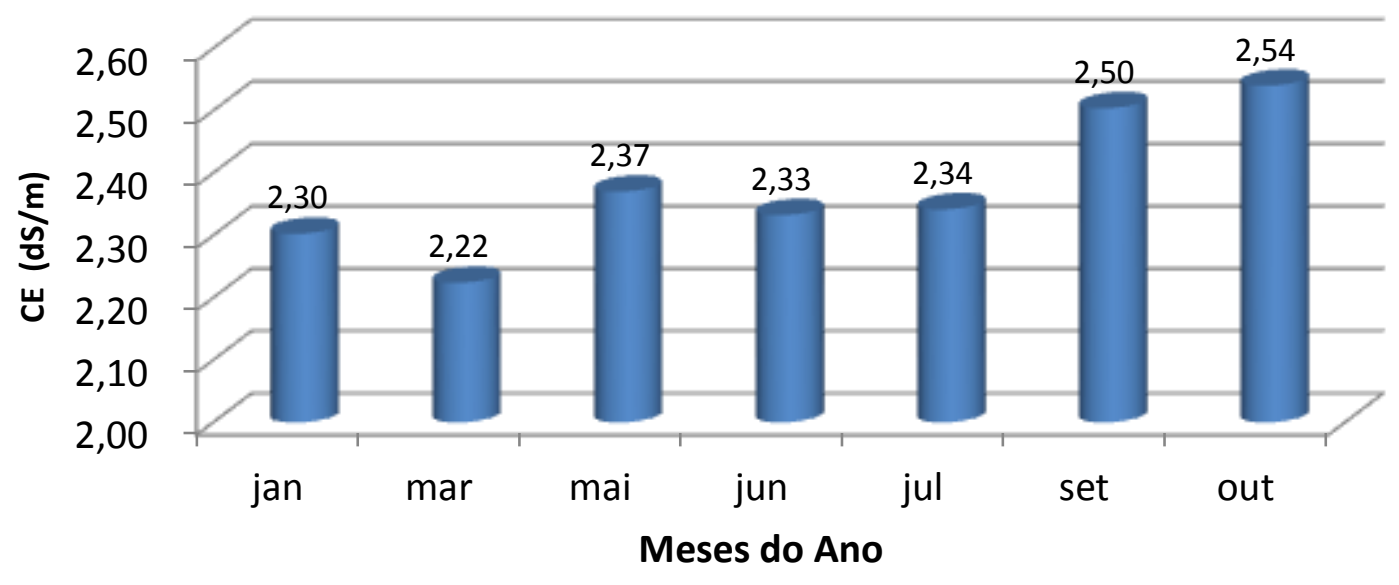

Fonte: Os autores (2012).

A análise do controle de qualidade para medidas individuais da $C E$, representada pelo gráfico de Shewhart, com a determinação da linha media (LM), do Limite Superior de Controle (LSC) e Limite Inferior de Controle (LIC) estatístico é apresentado no gráfico 1. Para o $\mathrm{CE}$,a média encontrada nos 13 poços analisados, ao longo do ano de 2012, foi de 2,37dS $\mathrm{m}^{-1}$. Face ao número de observações, foi utilizada a regra de controle $\mathbf{1}_{25}$ onde percebe-se que duas das observações excederam os limites de controle $x \pm 2 \mathrm{DP}$, o poço $10 \mathrm{com}$ valor de CE igual a $2,53 \mathrm{dS} \mathrm{m}^{-1}$, e o poço $13 \mathrm{com}$ valor de CE igual a 2,30 dS $\mathrm{m}^{-1}$.Contudo, nenhum valor atingiu os Limite de Controle (LC) estatístico, conforme Westgard et al.(1981).

Gráfico 1- Controle de qualidade de Shewhart, das medidas individuais da condutividade elétrica (CE).Ano 2012.

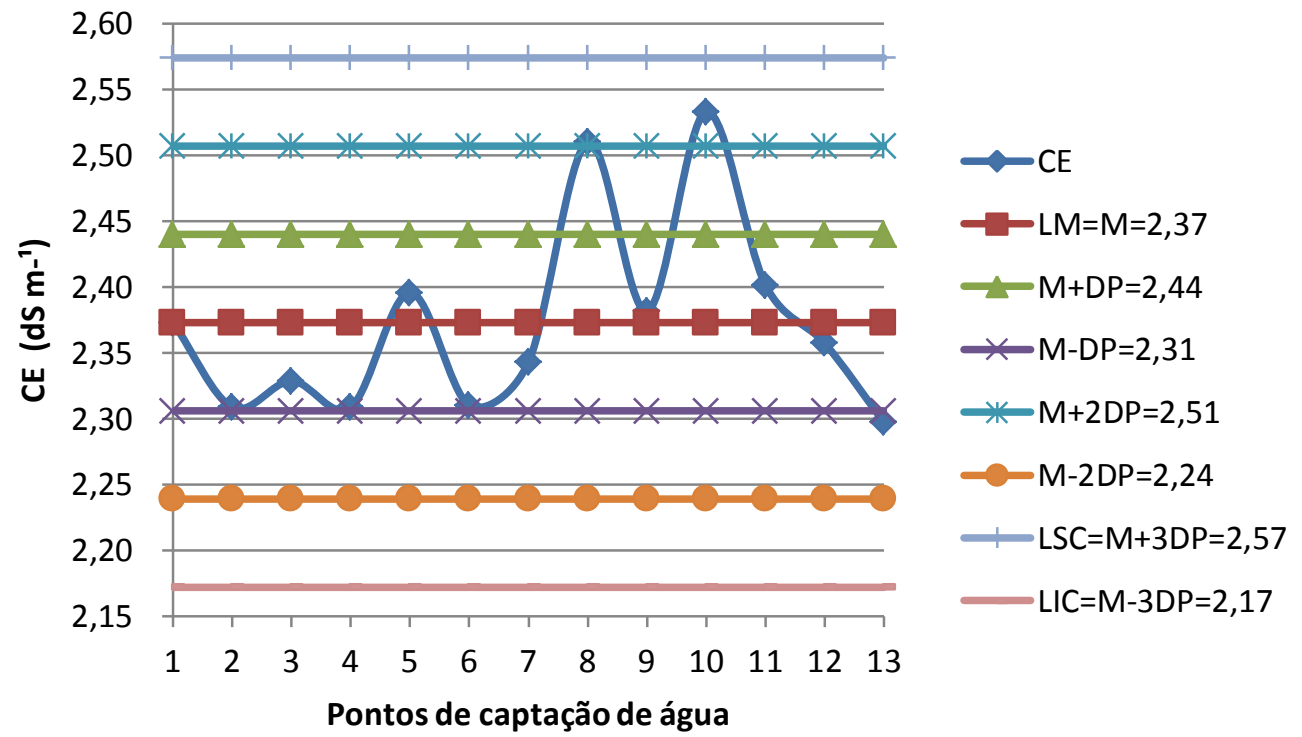

Fonte: Os autores (2012). 
NITRATO

O nitrato constitui-se na principal forma de nitrogênio associado à contaminação da água pelas atividades agropecuárias. Os fertilizantes nitrogenados são empregadosem grandes quantidades na agricultura moderna e o seu uso indiscriminado pode acarretar a contaminação, principalmente das águas subterrâneas. A quantidade excessiva de nitrato para o lençol freático é oriunda, sobretudo, de um desequilíbrio entre a quantidade, no solo, de nitrogênio, na forma amoniacal ou nítrica, e as necessidades da planta em assimilar esses teores.

A lixiviação do nitrato em áreas agrícolas é potencializada pelas propriedades físicas dos solos, pela quantidade de nitrato presente ou adicionada ao solo, pelas práticas agrícolas intensivas, pelas condições climáticas (pluviosidade) e pela elevada dotação de água empregada na irrigação. Na solução do solo, este elemento fica muito propenso ao processo de lixiviação e ao longo do tempo pode haver considerável incremento de seus teores em águas subterrâneas, com possíveis riscos potenciais, diretos ou indiretos, à saúde humana, considerando um cenário de exposição, como no caso da irrigação. O valormáximo permitido, desses teores, em água subterrânea, corresponde a $10.000 \mu \mathrm{g} \cdot \mathrm{L}^{-1}\left(\mathrm{~N}-\mathrm{NO}_{3}{ }^{-}\right)$, de acordo com anexo II da Resolução CONAMA 420/2009.

Os valores de nitrato nos poços da fazenda Dinamarca variaram de 17150 a $10070 \mu \mathrm{g} . \mathrm{L}^{-1}$ $\left(\mathrm{N}-\mathrm{NO}_{3}{ }^{-}\right)$, com valor médio de $13510 \mu \mathrm{g} \cdot \mathrm{L}^{-1}\left(\mathrm{~N}^{-\mathrm{NO}_{3}}{ }^{-}\right)$. Assim, verifica-se oselevados valores de $\mathrm{N}$ $\mathrm{NO}_{3}{ }^{-}$, que contribuem para contaminação das amostras de águas subterrâneas avaliadas, de acordo com anexo II da Resolução CONAMA 420/2009.

Segundo Ayers e Westcot (1999), para valores de até $5000 \mu \mathrm{g} \cdot \mathrm{L}^{-1}\left(\mathrm{~N}-\mathrm{NO}_{3}{ }^{-}\right)$de nitrato, na água de irrigação, não há restrição, e de 5000 a $30000 \mu \mathrm{g} \cdot \mathrm{L}^{-1}\left(\mathrm{~N}-\mathrm{NO}_{3}{ }^{-}\right)$a restrição é de ligeira amoderada.Por sua vez, os valores limites de nitrato devem ser, no máximo, de $10000 \mu \mathrm{g} . \mathrm{L}^{-1}$ (N$\mathrm{NO}_{3}{ }^{-}$) na água de irrigação para hortaliças,a fim de prevenir que não ocorram problemas de contaminação de águas subterrâneas e superficiais e, também, problemas de saúde aos consumidores de alimentos com excesso de nitrato(BRASIL,2001,2005). Portanto, quanto ao nitrato, todas as amostras de água analisadas, no presente trabalho, possuem restrição de ligeira a moderada, considerando as recomendações de Ayers e Westcot (1991), e acima dos valores máximos permitidos, para a água doce, quesão de $10,0 \mathrm{mg} \cdot \mathrm{L}^{-1}\left(\mathrm{~N}_{-} \mathrm{NO}_{3}{ }^{-}\right)$pela legislação brasileira Res.n $n^{\circ}$ 557/2005 (CONAMA), e Portaria 2.914/2011, sendo imprópria para o consumo humano, caso não sejam realizados certos procedimentos, para baixar os níveis de nitrato.Para a irrigação, na cultura do melão, normalmente a água de irrigação por gotejamento não entra em contato com o fruto e a absorção da quantidade desse nutriente pelo sistema radicular está vinculada aos estágios de desenvolvimento da cultura, onde o nível de tolerância pode variar entre as fases de desenvolvimento e, consequentemente, a sensibilidade do cultivo ao nitrato (MAAS;HOFFMANN, 1977; MAAS, 1990).

Para Almeida (2010), apesar das restrições das normas, não se deve considerar como necessariamente negativa a presença de Nitrato nas águas de irrigação, e sim positivamente desde que possa supor como uma adubação da unidade de fertilizante nitrogênio. Assim, a quantidade de fertilizantes nitrogenados que se aplique aos cultivos irrigados com água, a qual contenha níveis elevados de nitrato, deve-se reduzir a uma quantidade deste elemento similar à aplicada com a irrigação.

Os valores médios de nitrato da água coletada e analisada, nos poços tubulares, ao longo do ano de 2012, podem ser visualizados na Figura 3. Os maiores valores médios de nitrato foram 
encontrados nos meses de setembro eoutubro, 14120 e $13970 \mu \mathrm{g} \cdot \mathrm{L}^{-1}\left(\mathrm{~N}-\mathrm{NO}_{3}{ }^{-}\right)$, respectivamente, época de maior demanda de água da cultura do melão, consequentemente maior lixiviação de corretivos e fertilizantes. Os menores valores de nitrato ocorreram nos meses de janeiro e março, provavelmente pela diluição do nitrato em virtude de precipitações ocorridas entre dezembro de 2011 e fevereiro de 2012. Em todos os meses analisados os valores de nitratoencontrados nos poços tubulares, estão acima do valor máximo permitidos que é de $10,0 \mathrm{mg} \cdot \mathrm{L}^{-1}\left(\mathrm{NO}_{3}{ }^{-} \mathrm{N}\right)$ pela legislação brasileira Res.n³57/2005 (CONAMA) e pela Portaria MS 2.914/2011. O anexo II da Res. $n^{\circ} 420 / 2009$ (CONAMA), estabeleceo valor máximo permitido de $10000 \mu \mathrm{g} . \mathrm{L}^{-1}\left(\mathrm{~N}^{-\mathrm{NO}_{3}}{ }^{-}\right.$).

A variabilidade no valor de nitrato, entre estações secas e chuvosas, também foi observada por Franca et al (2006), ao analisarem a qualidade das águas de uma bateria de poços tubulares, que abastece o município de Juazeiro do Norte, CE. Durante o período seco, a concentração média de nitrato da bateria de poços estudados variou de $15600 \mu \mathrm{g} . \mathrm{L}^{-1} \mathrm{Na} 800 \mu \mathrm{g} . \mathrm{L}^{-1}$ $\mathrm{N}$. Durante o período chuvoso, a concentração média de nitrato variou entre $5800 \mu \mathrm{g} \cdot \mathrm{L}^{-1} \mathrm{Na}$ $300 \mu \mathrm{g} \cdot \mathrm{L}^{-1} \mathrm{~N}$.

Andrade (2009) investigou durante três anos as flutuações nas concentrações de nitrato em quatro poços subterrâneos no Perímetro Irrigado do Baixo Acaraú, CE. Dois desses poços estavam inseridos nos campos irrigados (P1 e P2) e dois (P3 e P4) em áreas de mata nativa. No período inicial do estudo, dois anos após a implantação do Distrito de Irrigação, os teores de nitrato nas águas eram semelhantes em todos os poços monitorados. Seis meses depois, as concentrações de $\mathrm{P} 1$ e $\mathrm{P} 2$ passaram a ser superiores àquelas registradas nos poços inseridos na área de mata nativa. As maiores concentrações de nitrato nas águas de P1 e P2 ocorreram nos meses de março e maio de 2007, com teores entre 19400 e $17100 \mu g . \mathrm{L}^{-1}\left(\mathrm{~N}-\mathrm{NO}_{3}\right.$ ).respectivamente, estando $94 \%$ e $71 \%$ acima do limite máximo legal aceitável. Uma vez que o efeito acumulativo foi constatado somente nos poços inseridos na área irrigada, acredita-se ser o mesmo decorrente da aplicação dos fertilizantes minerais nitrogenados e orgânicos, em doses de $80 \mathrm{~kg} \mathrm{~N} \mathrm{ha}^{-1}$ e $430 \mathrm{~kg} \mathrm{~N} \mathrm{ha}^{-1}$, respectivamente. Este fato expressa a necessidade de mudanças no manejo da irrigação adotado nas áreas do referido Distrito de Irrigação.

Figura 3 - Valores médios de nitrato $\left(\mathrm{NO}_{3}^{-}-\mathrm{N}\right)$ dos poços, ao longo do ano de 2012, e o valor máximo permitido (VMP) pela legislação brasileira.

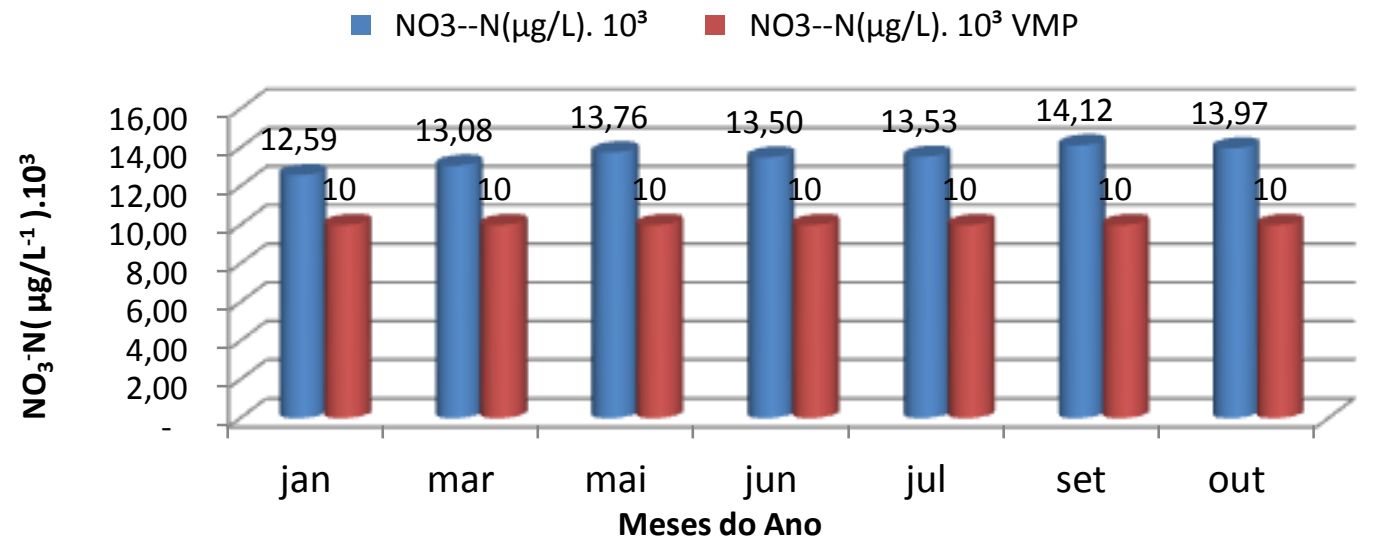

Fonte: Os autores (2012). 
O gráfico 2 representa o controle de qualidade de Shewhart, para as concentrações individuais de nitrato, com a determinação da linha media (LM), do Limite Superior de Controle (LSC) e do Limite Inferior de Controle (LIC) estatístico. Para a concentração de nitrato, a média encontrada nos 13 poços analisados, no decorrer do ano de 2012 , foi de $13,51.10^{3} \mu \mathrm{g} \cdot \mathrm{L}^{-1}\left(\mathrm{~N}-\mathrm{NO}_{3}{ }^{-}\right.$ ).Foi utilizada a regra de controle $1_{2 s,}$ onde se percebe graficamente que nenhuma das concentrações excede os limites de controle $x \pm 2 \mathrm{DP}$, consequentemente nenhum valor atingiu os Limite de Controle (LC) estatístico, conforme Westgard et al. (1981), evidenciando um resultado satisfatório da qualidade das análises de nitrato.

Gráfico 2 - Controle de qualidade de Shewhart, das medidas individuais das concentrações de nitrato

(NO3- -N ).Ano 2012

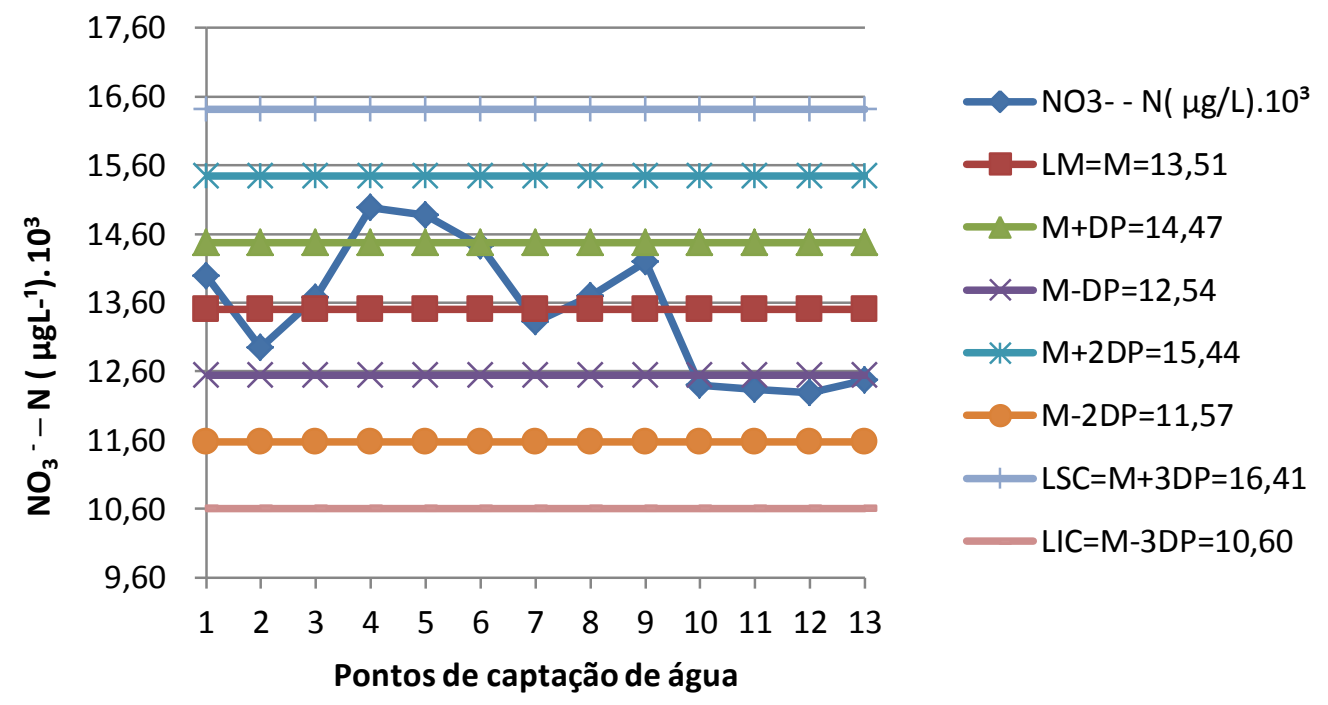

Fonte: Os autores (2012).

\section{POTENCIAL HIDROGENIÔNICO}

O potencial hidrogeniônico $(\mathrm{pH})$ é um parâmetro indicativo do grau de acidez ou alcalinidade do meio. No caso das águas de irrigação, o pH normal é entre 6,5 e 8,4. Os valores de $\mathrm{pH}$ nos poços da fazenda Dinamarca variaram de 7,71 a 7,14, com valor médio de 7,40 (Tabela 3). O coeficiente de variação dos dados foi de $1,71 \%$, sendo considerado baixo, de acordo com Pimentel-Gomes (1985).

Os valores de $\mathrm{pH}$ encontrados na maioria das amostras de águas analisadas ficou entre 6,5 e 8,5. Normalmente o valor do pH é influenciado pela dissolução do ácido carbônico ou pelo intemperismo de rochas e da erosão de áreas agrícolas com uso de corretivos e fertilizantes (FRANCA et al., 2006). A legislação brasileirade acordo com a Portaria 2.914/2011, estabelece valores de $\mathrm{pH}$ entre 6,5 e 8,5 para águas destinadas ao consumo humano, e entre 6,0 e 9,0 para todas as classes de água doce. Para a irrigação, o limite de tolerância do $\mathrm{pH}$, de acordo com a classificação de Ayers e Westcot (1999), está entre 6,5 e 8,4. Essa faixa de pH está de acordo com limites de $\mathrm{pH}$ das amostras analisadas no presente trabalho. 
Observa-se, na Figura 4 que os valores médios de $\mathrm{pH}$ durante o período da pesquisa, não apresentaram grandes oscilações. O maior valor de $\mathrm{pH}$ foi encontrado no mês outubro, 7,50, época de maior demanda de água da cultura do melão, conseqüentemente maior lixiviação de corretivos e fertilizantes.Os menores valores de $\mathrm{pH}$ foram encontrados entre maio e junho, período de "descanso" do solo (pousio)para a recuperaçãoda sua bioestrutura e, por conseguinte, para sua fertilidade.

Observa-se, na Figura 4, que os valores médios de $\mathrm{pH}$ durante o período da pesquisanão apresentaram grandes oscilações. O maior valor de $\mathrm{pH}$ foi encontrado no mês de outubro, 7,50, época de maior demanda de água da cultura do melão, consequentemente maior lixiviação de corretivos e fertilizantes.

Pequena variabilidade no valor do pH entre estações secas e chuvosas também foi observada por Franca et al (2006), ao analisarem a qualidade das águas de uma bateria de poços tubulares, que abastece o município de Juazeiro do Norte (CE). Durante o período seco, o valor médio máximo de $\mathrm{pH}$ encontrado foi igual a 7,6, e o pH médio mínimo foi igual a 6,2. No período chuvoso, o valor médio máximo de pH encontrado foi igual 7,4 e o mínimo foi igual a 6,2.

Figura 4 - valores médios de pH ao longo do ano de 2012

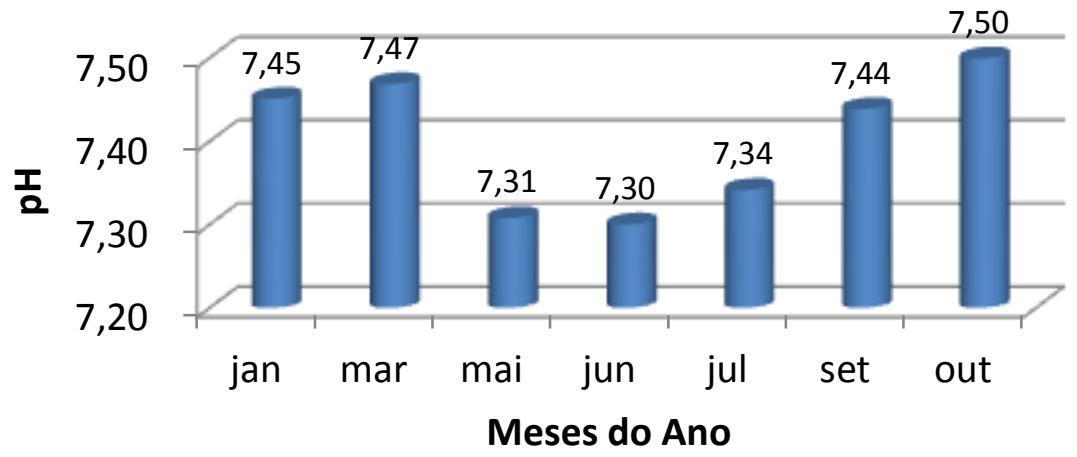

Fonte: Os autores (2012).

A análise do controle de qualidade das amostras de $\mathrm{pH}$, através do gráfico de Shewhart, com a determinação da linha media (LM), do Limite Superior de Controle (LSC) e Limite Inferior de Controle (LIC) estatístico é apresentado noGráfico 3. Para o pH a média encontrada nos 13 poços analisados, ao longo do ano de 2012 , foi de 7,40 . Foi utilizada a regra de controle $1_{2 s}$ ode se percebe que nenhumdos valores de $\mathrm{pH}$ excede os limites de controle $x \pm 2 \mathrm{DP}$, consequentemente nenhum valor atingiu os Limite de Controle (LC) estatístico, conforme Westgard et al.(1981), caracterizando resultados satisfatórios da qualidade das analises de $\mathrm{pH}$. 
Gráfico 3 - Controle de qualidade de Shewhart, das medidas individuais do pH.Ano 2012

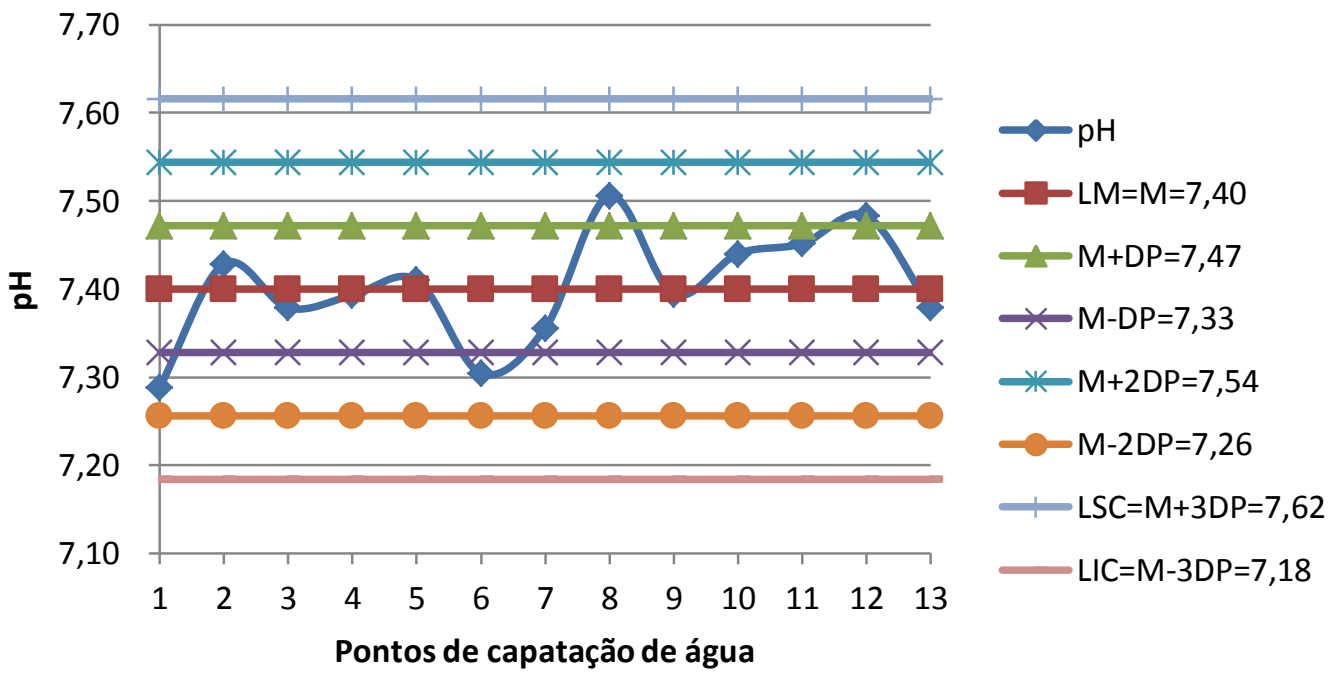

Fonte: Os autores (2012).

\section{CONCLUSÕES}

Através dos resultados analíticos dos parâmetros avaliados e dos critérios estabelecidos para a sua classificação, de acordo com as Normas vigentes, conclui-se que a salinidade das águas dos poços da fazenda Dinamarca expressos pela condutividade elétrica, foi classificada, quanto à restrição e uso, como de ligeira a moderadamente salina. As concentrações de nitrato, nas águas foram elevadas, podendo ser usadas com restrição de ligeira à moderada na irrigação. Os valores médios de $\mathrm{pH}$ apresentaram variações aceitáveis para utilização da água na irrigação no período estudado.

Em relação a todos os parâmetros analisados, se sobressai os elevados teores de nitrato com valores acima do máximo permitido (VMP) pela legislação brasileira para o consumo humano, de acordo com a Portaria MS 2.914/2011, e da Resolução CONAMA 357/2005, referente à classificação dos corpos de água, e do anexo II da Resolução CONAMA 420/2009, referente aos valores de referências para água subterrâneas. No entanto, a qualidade da água pode ser melhorada, com o estabelecimento de um manejo adequado da irrigação, com vistas ao controle da salinização na área levando-se em conta que a cultura do melão é classificada como moderadamente sensível à salinidade.

\section{REFERÊNCIAS}

ALMEIDA, O. A. de. Qualidade da água de irrigação. Cruz das Almas: Embrapa mandioca e Fruticultura, 2010. Disponível

em:<http://www.cnpmf.embrapa.br/publicacoes/livro/livro_qualidade_agua.pdf >. Acessoem: 10 dez. 2012.

AMERICAN PUBLIC HEALTH ASSOCIATION (APHA).Standard methods for theExamination of Water andWastewater.20 ed. United StatesofAmerica , 2005. 
ANDRADE, E. M. A irrigação e suas implicações sobre o capital natural em regiões áridas e semiáridas: uma revisão. Revista CERES, Viçosa, v. 56, n. 4, p. 390-398, 2009.

ANDRADE, E. M.; AQUINO, D. N.; CRISÓSTOMO, L. A.; RODRIGUES, J. O.; LOPES, F.B. Impacto da lixiviação de nitrato e cloreto no lençol freático sob condições de cultivo irrigado. Ciência Rural, Santa Maria, v.39, n.1, p.88-95, 2009

AYRES, R.S.; WESTCOT, D.W. A qualidade da água na agricultura. 2.ed. Campina Grande: UFPB, 1999. 153p.(Estudos FAO, irrigação e Drenagem 29, revisado 1).

BEN-HUR, M.; LI, F.H.; KEREN, R.; RAVINA, I.; SHALIT, G. Water and salt distribution in a field irrigated with marginal water under high water table conditions. Soil Science American Journal, v. 65, p.191-198, 2001.

BRASIL. Ministério do Meio Ambiente. Resolução CONAMA № 357 de 17 de março de 2005. Brasília-DF: 2005. 23p.Dispõe sobre a classificação dos corpos de água e diretrizes ambientais para o seu enquadramento. Brasília, 2005, p.58-63.

BRASIL. Ministério do Meio Ambiente. Resolução CONAMA № 420 de 30 de dezembro de 2009. Brasília-DF: 2009. 18p.Dispõe sobre critérios e valores orientadores de qualidade do soloquanto à presença de substâncias químicas e estabelece diretrizes para o gerenciamento ambiental deáreas contaminadas por essas substâncias em decorrência de atividades antrópicas. Brasília, 2009, p.81-84.

BRASIL. Ministério da Saúde.Portaria № 2.914, de 12 de dezembro de 2011. Brasília-DF: 2011. 34p. Dispõe sobre os procedimentos de controle e de vigilância da qualidade da água para consumo humano e seu padrão de potabilidade. Disponível em:

<http://www.ms.gov.br/conama/. Acesso em: 10 dez.2012.

CARMO FILHO, F.; OLIVEIRA, O. F. Mossoró: um município do semi-árido nordestino, caracterização climática e aspecto florístico. Mossoró: ESAM, 1995. 62p. (Coleção Mossoroense, Série B).

RIO GRANDE DO NORTE. Empresa de pesquisa Agropecuária do Rio Grande do Norte S/A.(EMPARN)Previsão Climática. Disponível em:http://189.124.135.176/monitoramento/2012/acumulapr.htmAcesso em: 12 jan. 2012.

FRANCA, R. M.; FRISCHKORN, H.; SANTOS, M. R. P.; MENDONÇA, L. A. R.; BESERRA, M. C. Contaminação de poços tubulares em Juazeiro do Norte-CE.Engenhariasanitária e ambientalv. 2, n.1, p. 92-102, 2006.

FRISTZSONS, E.; RIZZI, N.; BITTENCOURT,A.V.L.; MANTOVANI, L. E. Estudo do impacto de contaminação por nitrogênio numa bacia hidrográfica cárstica. Boletim Paranaense deGeociências, n. 49, p. 39-52, 2001.

GARCÍA-GARIZÁBAL, I.; J. CAUSAPÉ, J.; R. ABRAHAO, R. Nitrate contamination and its relationship with flood irrigation management.Journal of Hydrology, v. 442, p. 15-22, 2012

GALLOWAY, J.N., TOWNSEND, A.R., ERISMAN, J.W., BEKUNDA, M., CAI, Z.C., FRENEY, J.R., MARTINELLI, L.A., SEITZINGER, S.P., SUTTON, M.A. Transformation of the nitrogen cycle: recent trends, questions, and potential solutions. Science, v.320, p.889-892, 2008

GOOGLE, Programa Google Earth, 2012 
HASSANLI, A. M., AHMADIRAD, S., BEECHAM, S. Evaluation of the influence of irrigation methods and water quality on sugar beet yield and water use efficiency.Agricultural Water Management, n.97, p.357-362, 2009.

LILLIEFORS, H.W. On the Kolmogorov-Smirnov test for normality with mean andvariance unknown.Journal of the American Statistical Association, v. 62, n. 399-402, 1967.

MAROUELLI, W.A.; MEDEIROS, J.F.; PINTO, J.M.; SILVA, W.L.C. Irrigação.In: SILVA, H.R.; COSTA, N.D. Melão, produção aspectos técnicos. Brasília: Embrapa Hortalíças, Embrapa Semiárido, Embrapa Informação Tecnológica, 2003. p.51-68

MAAS, E. V.; HOFFMAN, G. J. Cropsalttoleranceassessment. Journal of the Irrigation and Drainage Division, ASCE,v.103, n.2, p.115-134, 1977.

MAAS, E. V. Crop salt tolerance. In: Agricultural salinity assessmentand management. In: TANJI, K.K. (Ed). New York, American Society of Civil Engineers, 1990. p. 262-304.

MEDEIROS, J. F. de; NASCIMENTO, I.B.; COSTA, M. da C.; SCALOPPI, E.J. Produção de melão sob diferentes laminas de água com dois níveis de salinidade. Horticultura Brasileira, Brasília, DF, v.18, p.612-614, 2000. Suplemento.

MEDEIROS, J. F, de; LISBOA, R. de A.; OLIVEIRA, M. de. Caracterizaçãodas águas subterrâneas usadas para irrigação na área produtora de melão da Chapada do Apodi. Revista Brasileira de Engenharia Agrícola e Ambiental, v. 7, p.469-472, 2003

MEDEIROS, J. F. de; NASCIMENTO, L. B. do; GHEYI, H.R. Manejo do solo-água-planta em áreas afetadas por sais. In: GHEYI, H.R.; SILVA, DIAS, N. da S.; LACERDA, C. F. de. Manejo da salinidade na agricultura: estudos básicos e aplicados. Fortaleza: INCT Sal, 2010, p.280-302.

MONTGOMERY, D. C. Introdução aocontrole estatístico da qualidade.Tradução: Farias, A. M. L., Flores,V. R. L. F., Laurencel, L. C. 4. ed.Rio de Janeiro: Editora LTC, 2004.

OLIVEIRA, M. DE; MAIA, C. E. Qualidade físico-química da água para irrigação em diferentes aquíferos na área sedimentar do Estado do Rio Grande do Norte. Revista Brasileira de Engenharia Agrícola e Ambiental, v. 2, p. 42-46,1998

PEREIRA, O. J. MATIAS FILHO, J.; ANDRADE, E. M. Variação do teor de sais no solo irrigado por aspersão e ação da chuva na sua lixiviação. Ciência Agronômica, v.17, p.61-65, 1986

PIMENTEL-GOMES, F. Curso de Estatística Experimental. 12. ed. Piracicaba:Livraria Nobel, 1985. $467 p$.

REIS, C. F.; VILAS BOAS, M. A.; MERCANTE, E.; HERMES, E.; REISDORFER, M. Avaliação da qualidade da água para irrigação em Salto doLontra - PR.Engenharia Ambiental, Espírito Santo do Pinhal, v. 8, n. 1, p. 069-078, 2011.

RODRIGUES, J. O.; ANDRADE, E. M. de; CRISÓSTOMO, L. A.; TEIXEIRA, A. dos S. Modelos da concentração iônica em águas subterrâneas no Distrito de Irrigação Baixo Acaraú.

RevistaCiênciaAgronômica, v.38, n.4, p.360-365, 2007.

SHEWHART, W. Economic control of quality of manufactured product. New York: D. Van NostrandCompany, 1931. 501p.

SILVA, A. B. da.Hidrogeologia de meios cársticos Disponível em:

<http://www.nehma.ufba.br/cursos/apostilas_monografia/Graduacao/LIVRO texto.pdf.> Acesso em:10 jan. 2013. 
SILVA, F. DE A. S. E, AZEVEDO, C. A. V. de. Versão do programa computacionalAssistat para o sistema operacional Windows. Revista Brasileira de Produtos Agroindustriais, Campina Grande, v.4,n.1,p7178,2002 .

SOUZA NETO, E. R. et al. Produção de melão irrigado com águas de diferentes níveis de salinidade nas diferentes fases da cultura. RevistaCaatinga, v. 16, n. 01/02, p. 39-45, 2003.

SRINIVASAN, J. T., REDDY, V. R. Impact of irrigation water quality on human health: a case study in India. Ecological economics, Hyderabad, n.101, p.2800-2807,2009.

WESTGARD, J. O.; BARRY, P. L.; HUNT, M. R.A Multi-Rule Shewhart Chart for Quality Control in Clinical Chemistry.Clinical Chemistry, v. 27, n.3, p.493-501, 1981. 